\title{
Alkali Halide Microstructured Optical Fiber for X-ray Detection
}

\author{
S.L. DeHaven ${ }^{1 a)}$, R.A. Wincheski ${ }^{1 b)}$, and S. Albin ${ }^{2 c)}$ \\ ${ }^{1}$ NASA Langley Research Center, Hampton, VA 23681 \\ ${ }^{2}$ Norfolk State University, Norfolk, VA 23504 \\ ${ }^{a)}$ Corresponding author: stanton.1.dehaven@nasa.gov \\ b)russel.a.wincheski@nasa.gov \\ ${ }^{c)}$ salbin@nsu.edu
}

\begin{abstract}
Microstructured optical fibers containing alkali halide scintillation materials of $\mathrm{CsI}(\mathrm{Na}), \mathrm{CsI}(\mathrm{Tl})$, and $\mathrm{NaI}(\mathrm{Tl})$ are presented. The scintillation materials are grown inside the microstructured fibers using a modified BridgmanStockbarger technique. The x-ray photon counts of these fibers, with and without an aluminum film coating are compared to the output of a collimated CdTe solid state detector over an energy range from 10 to $40 \mathrm{keV}$. The photon count results show significant variations in the fiber output based on the materials. The alkali halide fiber output can exceed that of the CdTe detector, dependent upon photon counter efficiency and fiber configuration. The results and associated materials difference are discussed.
\end{abstract}

\section{INTRODUCTION}

$\mathrm{X}$-ray detectors have developed over time for different energy ranges and applications. There are many types of x-ray detectors including film, ionization chambers, Geiger-Mueller counters, scintillation, and semiconductors. The primary types for typical NDE use are scintillator and semiconductor, both in research and commercial applications.

The two primary categories of scintillation detectors are organic and inorganic. Organic types are based on carbon-hydrogen molecules which emit light from interaction with $\mathrm{x}$-rays. Inorganic scintillators are composed of compounds of inorganic materials, grown in some form of crystals which emit light from interaction with $\mathrm{x}$-rays. Semiconductor detectors generate current when $x$-rays interact with the material. The solid state detectors have several benefits compared to scintillators but need an evacuated space behind a beryllium window and require cooling for dark noise considerations [1].

The inorganic scintillators considered in this work are $\mathrm{NaI}(\mathrm{Tl}), \mathrm{CsI}(\mathrm{Tl})$, and $\mathrm{CsI}(\mathrm{Na})$ which are well known and extensively used alkali halide inorganic scintillators. These scintillators typically use a large crystal directly coupled to a photodetector for $\mathrm{x}$-ray detection and measurement. $\mathrm{NaI}(\mathrm{Tl})$ is the standard inorganic scintillator from which all others are compared [2].

A conventional fiber for $\mathrm{x}$-ray detection is plastic PMMA scintillating fiber. The plastic fiber uses an organic scintillator mixed with a polymer as the core. The plastic has relatively high absorption losses for visible light which requires the core to be 1000 microns in diameter [3]. 
More recent work in scintillating fibers is the introduction of organic scintillators into quartz microstructured optical fiber [4, 5]. These fibers operate on total internal reflection. Other fibers have been used which contain scintillation (radioluminescent) core glass as part of scintillating glass fiber optical faceplates [6]. These optical fibers are limited by the numerical aperture which results in low light transmission efficiency.

The novel approach presented in this paper is combining inorganic alkali halide scintillators with microstructured optical fiber to form an x-ray detector. The alkali halide scintillators are grown inside the microstructured fibers quartz matrix. The light guiding is a combination of total internal reflection and cladding mode transmission, which differs from conventional fibers. This combination of light guiding mechanisms significantly increases the transmission efficiency.

A solid state CdTe x-ray detector is used to characterize the x-ray tube photon vs. energy over an energy range from 10-40 keV and compared with the optical fibers output. The geometry of the microstructured optical fiber is shown in Figure 1. Each inclusion, or hole, is 2.5 microns in diameter with 138 inclusions in the fiber. The outside diameter of the fiber is 125 microns, which allows connection to standard fiber optics.

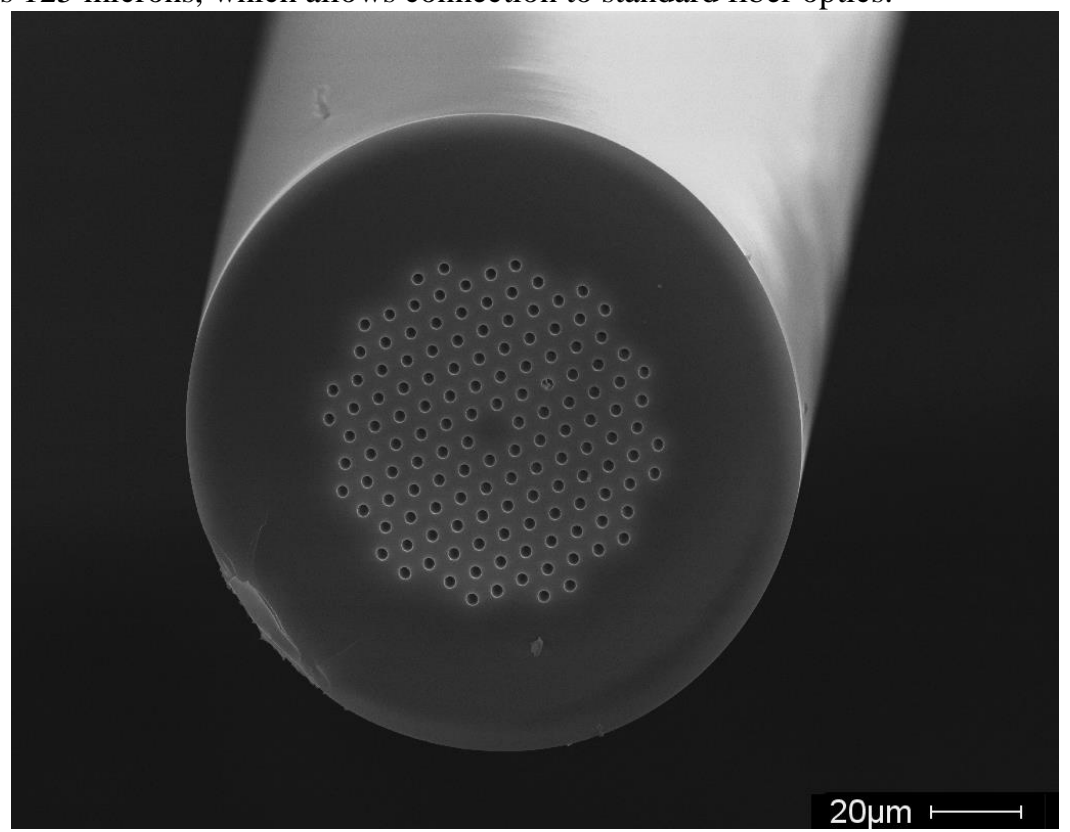

FIGURE 1. Shown is a SEM image of inclusions, or holes, in a microstructured optical fiber taken at $30 \mathrm{keV}$. The inclusions extend along the length of the fiber. Alkali halide scintillation materials are grown in these inclusions to form an $\mathrm{x}$-ray detecting optical fiber.

The fibers are compared with the CdTe detector in two configurations. The first has the fiber with no outside coating. This configuration shows high efficiencies from the combined light guiding mechanisms. The second shows significantly reduced efficiency when the fiber has a thin coating of aluminum on the outside surface.

\section{THEORY}

The x-ray detecting fiber microstructure contains 138 alkali halide fibers inside a quartz matrix. For light guidance using total internal reflection, this quartz matrix acts as a cladding. Total internal reflection is light guidance in two dimensions and relies on the difference in refractive index between the alkali halide and the quartz capturing and transmitting light in the alkali halide. This is the same mechanism used to describe the x-ray output of liquid filled and radioluminescent core fiber $[5,6]$.

The fibers presented in this work have the light generated and transmitted by the scintillator coupled to the microstructured quartz. This coupling is due to an air gap formed during the alkali halide crystal growth process and causes a three dimensional light guidance. Figure 2 shows a cross section of the alkali halide in the microstructured optical fiber with the air gap. Light is emitted from the end of the fiber with quartz and air. 


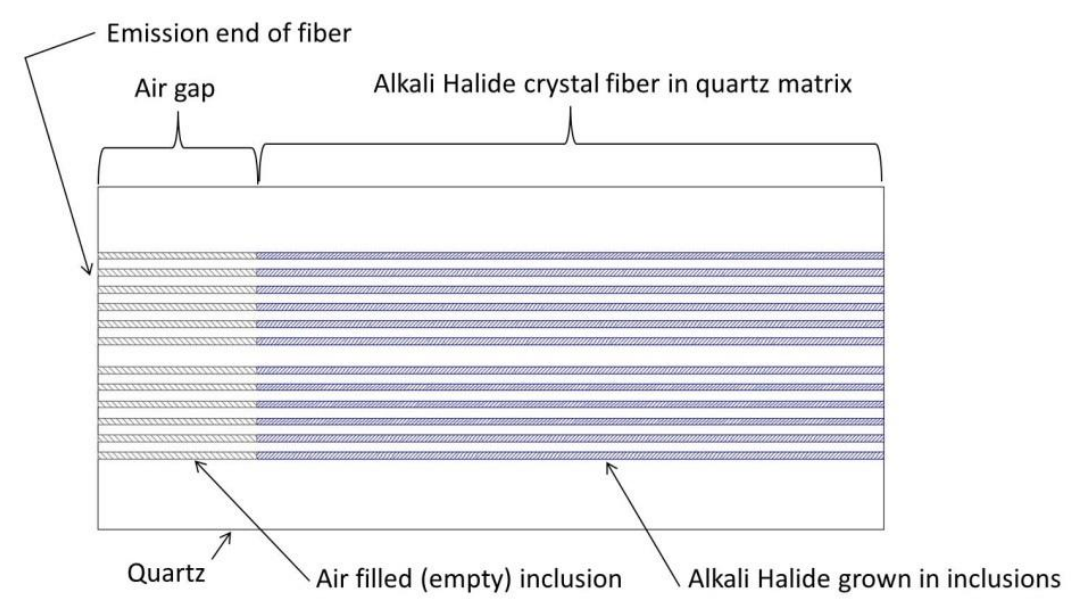

FIGURE 2. Cross section diagram of alkali halide in microstructured optical fiber showing the region where the fiber has an air gap. The scintillation light is emitted in the end of the fiber having air filled (empty inclusions). The air gap shown is formed during crystal growth.

$\mathrm{X}$-rays and UV light can produce visible light from the alkali halide scintillators. However, CsI(Na) does not fluorescence when exposed to UV light as noted in Table 1. This emission of light using UV allows examining the output of the fiber in a lab environment. Figure 3 shows CsI(Tl) microstructured optical fiber excited by a $254 \mathrm{~nm}$ UV lamp with the resulting $550 \mathrm{~nm}$ light emission from the fiber end. Table 1 shows the emission wavelength for each scintillator, UV fluorescence, and photons emitted per x-ray energy absorbed [2].

TABLE 1. The emission wavelength of scintillation light, whether the scintillator is fluorescent in the presence of UV light, and the photon emission per absorbed energy in $\mathrm{keV}$ is shown for the three alkali halides used [2]

\begin{tabular}{|c|c|c|c|}
\hline Alkali Halide & $\begin{array}{l}\text { Emission } \\
\text { Wavelength (nm) }\end{array}$ & $\begin{array}{l}\text { UV fluorescence } \\
\text { @ } 254 \mathrm{~nm}\end{array}$ & $\begin{array}{l}\text { Photon Emission } \\
\text { photons/keV }\end{array}$ \\
\hline CsI(Tl) & 550 & Yes & 62 \\
\hline $\mathrm{NaI}(\mathrm{Tl})$ & 415 & Yes & 36 \\
\hline $\mathrm{CsI}(\mathrm{Na})$ & 420 & No & 38 \\
\hline
\end{tabular}

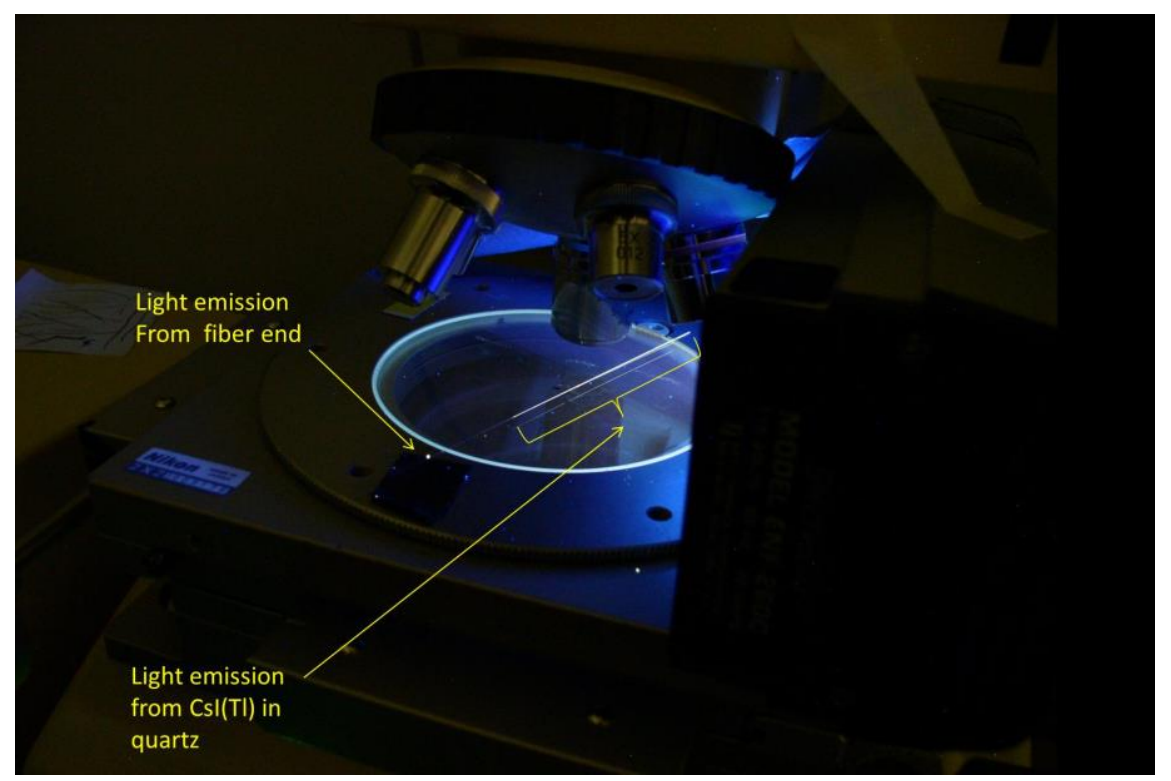

FIGURE 3. CsI(Tl) fiber after processing on a microscope stage. The $550 \mathrm{~nm}$ light is emitted from the $\mathrm{CsI}(\mathrm{Tl})$ fluorescence in the quartz using a $254 \mathrm{~nm}$ UV lamp. 
The energy range used in this work is $10-40 \mathrm{keV}$. Because of this lower energy range, all of the $\mathrm{x}$-ray interaction with alkali halide scintillators is considered absorption from the photoelectric effect. Absorption of the x-rays follows Beer's law along the axial fiber direction. Because of the high mass absorption coefficient of the alkali halide materials, less than $0.5 \mathrm{~mm}$ length is required to completely absorb the $\mathrm{x}$-rays [7]. The resultant absorbed $\mathrm{x}$ ray produces hundreds of photons, dependent upon the x-ray energy as shown in Table 1. A fraction of the emitted photons is captured in the scintillator with the remainder going through the quartz.

It is the guidance of the scintillation photons in the quartz optics which provides higher efficiency. To evaluate this theory, which is based on experimental observations, an aluminum coating is added to evaluate the effect on the optics and light guidance through the quartz. The aluminum coating on the quartz exterior causes a drastic reduction in the efficiency. The reduction in efficiency after applying the aluminum coating is from optical effects.

\section{EXPERIMENT}

The alkali halide fibers were prepared using a modified Bridgman-Stockbarger technique. A diagram of the furnace and experimental apparatus is shown in Figure 4. An ampoule holds the alkali halide material and base of the fiber in an inert gas chamber. The chamber and ampoule are raised to the melt zone of the furnace to allow capillary action to fill the microstructured optical fiber once the alkali halide has melted. Subsequently, the chamber is slowly lowered into the freeze zone of the furnace to grow alkali halide single crystals inside the microstructured optical fiber 2.5 micron diameter inclusions. The final fiber length is approximately $12 \mathrm{~cm}$.

Table 2 shows the upper and lower furnace melt and freeze zones with the traverse speed between the melt and freeze regions. The alkali halide melting temperatures were chosen for the bulk material [2]. The melt temperature was chosen to be $29^{\circ} \mathrm{C}$ above the melting point and the freeze zone $200{ }^{\circ} \mathrm{C}$ below the melt temperature. The freeze zone temperature was the lowest consistent temperature for the furnace arrangement used. The sodium iodide melt temperature was chosen to be $39{ }^{\circ} \mathrm{C}$ above the melting point with the freeze zone $200{ }^{\circ} \mathrm{C}$ lower. Similarly, this freeze zone temperature was the lowest consistent temperature for the furnace arrangement used. The transverse speed was found experimentally through a parametric study.

TABLE 2. The furnace set temperatures and translation speed along the $x$ direction as shown in Fig. 4 .

\begin{tabular}{llll}
\hline Alkali Halide & Upper zone & Lower zone & Translation \\
& Temperature $\left(\mathrm{T}_{\mathrm{m}}\right)$ & Temperature $\left(\mathrm{T}_{\mathrm{f}}\right)$ & Speed $(\mathrm{mm} / \mathrm{hr})$. \\
\hline $\mathrm{CsI}(\mathrm{Tl})$ & 650 & 450 & 35 \\
$\mathrm{NaI}(\mathrm{Tl})$ & 700 & 500 & 25 \\
$\mathrm{CsI}(\mathrm{Na})$ & 650 & 450 & 25
\end{tabular}

The inert gas chamber was evacuated between $2 \times 10^{-2}$ and $5 \times 10^{-2}$ Torr for 48 hours. Prior to melting, 1.1 atm of high purity argon gas was added to the gas chamber. The chamber was held at the melt temperature for 45 minutes before translation began down into the freeze zone. This downward direction of motion into the freeze zone causes an empty region at the upper portion of the fiber from the alkali halide density increasing on freezing.

Upon completing the growth processing, the $\mathrm{NaI}(\mathrm{Tl})$ and $\mathrm{CsI}(\mathrm{Na})$ fibers are placed in a dry box due to their hygroscopic nature. A CsI(Tl) fiber is shown in Figure 3 after processing on a microscope stage. The microscope allowed inspection of the alkali halide materials for single crystal formation.

The experimental technique used in previous work was applied for characterization of the $\mathrm{x}$-ray tube source and photon counting with the prepared alkali halide fibers [4]. The x-ray tube characteristic photons versus tube energy was plotted for tube voltages between $10 \mathrm{kV}$ to $40 \mathrm{kV}$ in $5 \mathrm{kV}$ increments. A $40 \mathrm{kV}$, $4 \mathrm{~W}$ silver anode $\mathrm{x}$-ray tube was the source with a piece of 30 micron aluminum foil placed at the exit as a low energy filter.

Figure 5 shows the experimental setup for $\mathrm{x}$-ray tube characterization, the scintillating fiber output using a photon counting unit, and scintillation fiber output using a photomultipler tube system. The CdTe x-ray spectrometer uses a collimator to collect the x-ray photons. When measuring output from the fiber, a photon counting unit is used for the $\mathrm{CsI}(\mathrm{Tl})$ fiber and a photomulitipler tube module for the $\mathrm{NaI}(\mathrm{Tl})$ and $\mathrm{CsI}(\mathrm{Na})$ fibers. 


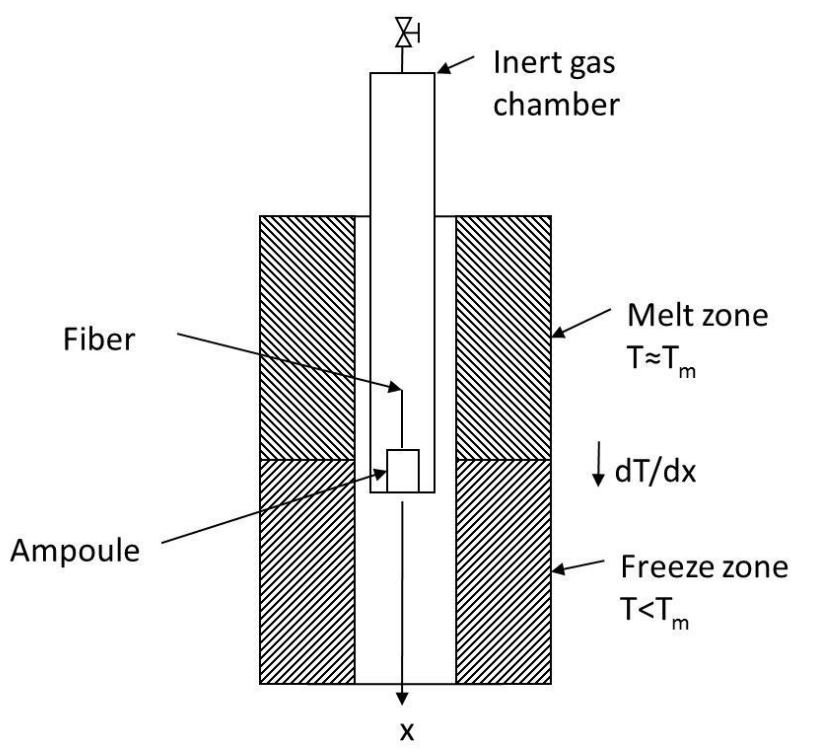

\section{Furnace Cross Section}

FIGURE 4. Diagram of the Bridgman-Stockbarger furnace showing the cross section with the features associated with alkali halide crystal grown inside microstructured optical fiber. The growth direction is along the $x$ axis.

The CdTe spectrometer operates with a multi-channel analyzer with a collimator having a 100 micron diameter tungsten aperture which is $5 \mathrm{~mm}$ thick followed by a 200 micron tungsten aperture $5 \mathrm{~mm}$ thick. The CdTe detector is $100 \%$ efficient at detecting $\mathrm{x}$-ray photons in the energy range between $10 \mathrm{keV}$ and $50 \mathrm{keV}$. The CdTe spectrometer operates with a computer to collect and store data.

The x-ray tube characterization was done prior to taking the fiber data. The CdTe detector energy scale was calibrated using an Am241 radioactive source. The collimator and $\mathrm{x}$-ray tube were carefully aligned to maximize $\mathrm{x}$ ray counts with the collimator placed $0.5 \mathrm{~cm}$ from the tube.

The prepared fibers were placed in the same location as the collimator while varying the $\mathrm{x}$-ray tube voltage. The $\mathrm{x}$-ray tube source current was set at the maximum value for all measurements. The fibers were coupled to either a photon counting module or a photomultiplier tube using a FC bare fiber optic coupler.

The $\mathrm{CsI}(\mathrm{Tl})$ fiber was coupled to a photon counting module with a quantum efficiency of approximately $59 \%$ at $550 \mathrm{~nm}$ wavelength. The $\mathrm{NaI}(\mathrm{Tl})$ and $\mathrm{CsI}(\mathrm{Na})$ fibers were coupled to a photomultiplier tube module with a quantum efficiency of approximately $25 \%$ at $420 \mathrm{~nm}$ wavelength. Table 3 shows the alkali halide scintillator used with the detector, light emission wavelength and detector quantum efficiency at this wavelength.

Signals from the photon counting module were sent to a counter with a count enabled voltage level set to $1.1 \mathrm{~V}$. Signals from the photomultiplier tube module went to a discriminator circuit with the threshold set to the lowest level. These pulses were sent to a counter with a count enabled voltage threshold set to $0.9 \mathrm{~V}$. The counts were accumulated over a five minute time period. The total pulse count was recorded for tube voltages between 10 and $40 \mathrm{kV}$ at $5 \mathrm{kV}$ increments.

TABLE 3. The alkali halides are listed with the photon counting detectors, scintillator output wavelengths and quantum efficiency at the output wavelength. The two types of detectors used with fiber were the photon counting module (PCM) and the photomultiplier tube (PMT).

\begin{tabular}{llll}
\hline Alkali Halide & $\begin{array}{l}\text { Photon } \\
\text { detector }\end{array}$ & $\begin{array}{l}\text { Light ouput } \\
\text { wavelength }(\mathrm{nm})\end{array}$ & $\begin{array}{l}\text { Detector quantum } \\
\text { efficiency }(\%)\end{array}$ \\
\hline $\mathrm{CsI}(\mathrm{Tl})$ & PCM & 550 & 59 \\
$\mathrm{NaI}(\mathrm{Tl})$ & PMT & 420 & 25 \\
$\mathrm{CsI}(\mathrm{Na})$ & PMT & 415 & 25
\end{tabular}




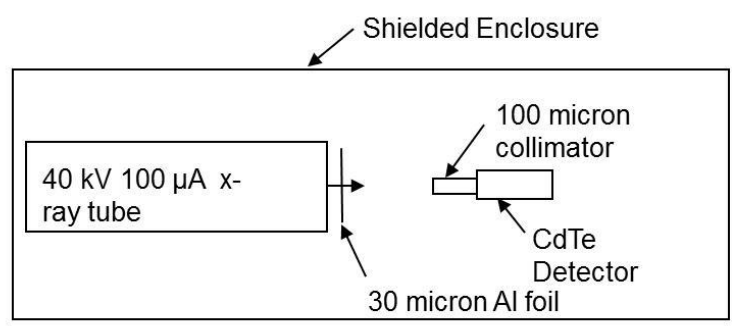

(a)

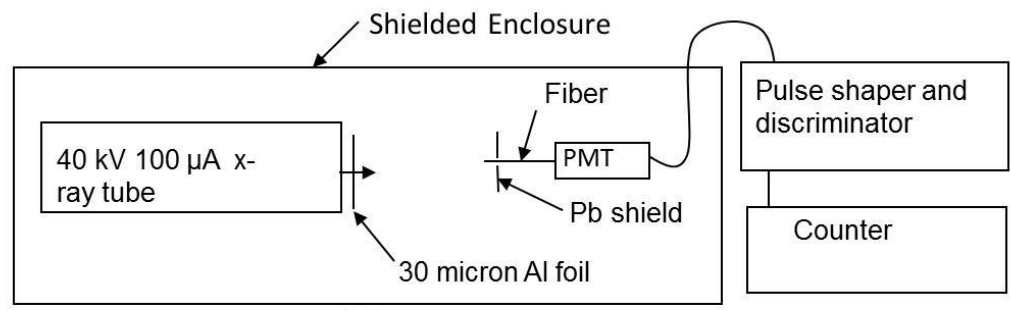

(b)

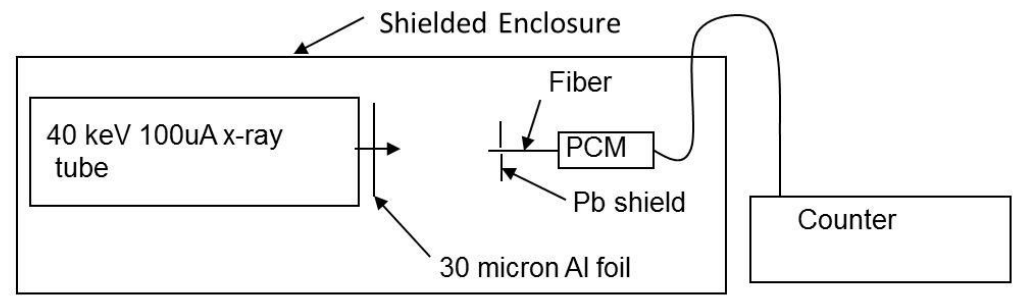

(c)

FIGURE 5. Experiment configuration for : (a) characterizing the $\mathrm{x}$-ray tube; (b) measuring the output of the $\mathrm{NaI}(\mathrm{Tl})$ and $\mathrm{CsI}(\mathrm{Na})$ fibers with a photmultiplier tube (PMT); (c) measuring the output of the CsI(Tl) fiber output with a photon counting module (PCM).

A blackout cloth was used over a leaded Plexigas three-sided enclosure. Power and cables were routed out the open side of the enclosure. The photomultiplier tube module was wrapped in lead sheet, the photon counting module and fiber couplers covered in lead plate to reduce noise.

The blackout cloth reduced stray photons while allowing easy access to the experiment. The room was partially darkened during operation to remove fluorescent fixture noise. Power for the electronics had a common ground.

An aluminum coating was applied to the fibers after taking the uncoated efficiency measurements and the measurements repeated. A thickness of approximately 0.1 micron of $99.99 \%$ pure aluminum was sputter coated on the outside of the processed fibers. The aluminum coating was applied using DC magnetron sputtering in argon. The argon pressure was $20 \times 10^{-3}$ Torr and the power was 350 watts.

\section{RESULTS AND DISCUSSION}

Figure 6 shows the $\mathrm{x}$-ray tube photon energy spectrum collected using the CdTe spectrometer with 100 micron collimator for tube characterization. A 30 micron thick sheet of aluminum foil was placed in front of the tube as a low energy filter. The collimator and fibers were positioned $0.5 \mathrm{~cm}$ from the tube end. Data was taken over five minute intervals. 


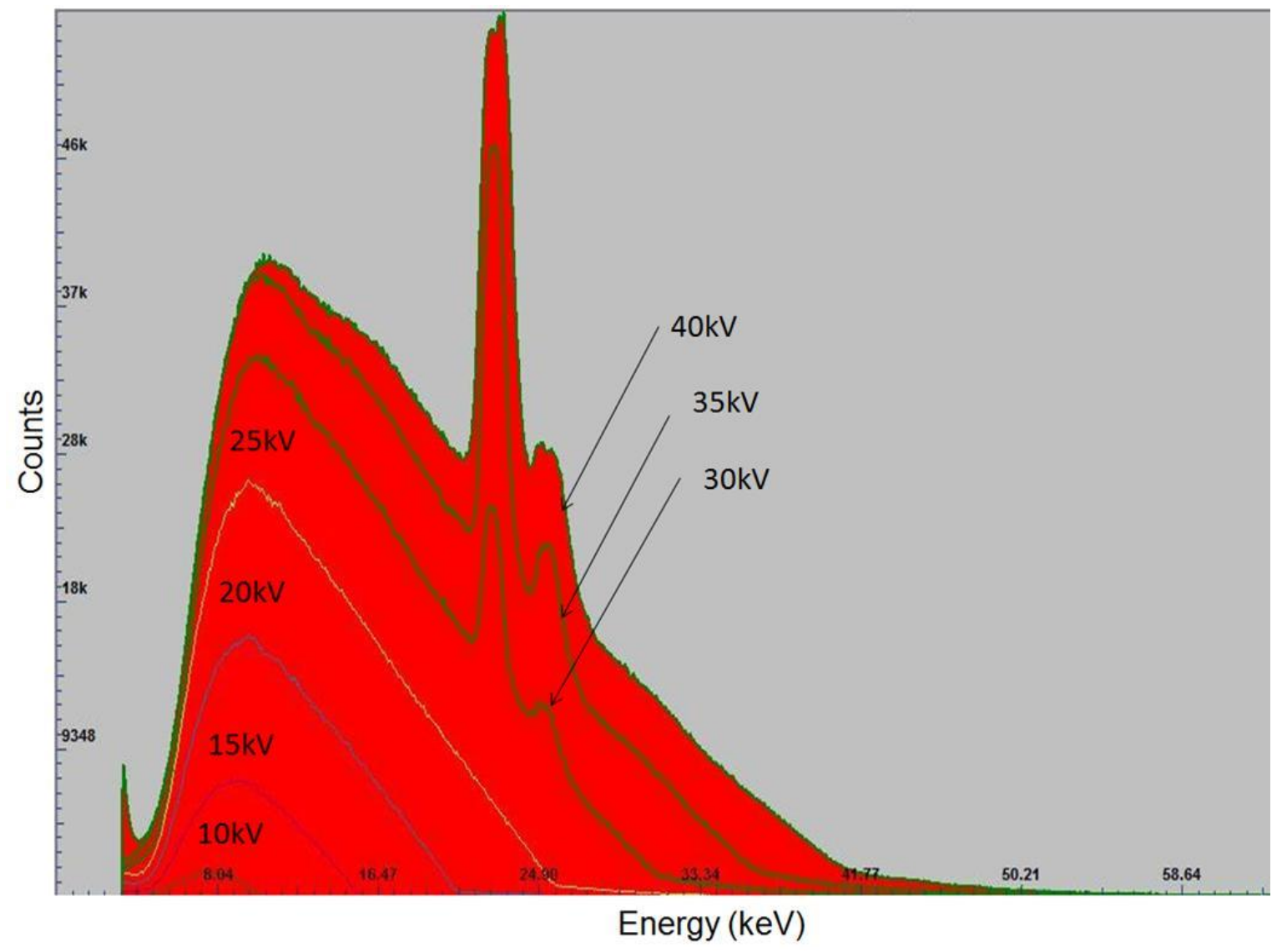

FIGURE 6. Characterization for $4 \mathrm{~W} 40 \mathrm{kV}$ silver anode $\mathrm{x}$-ray tube showing number of photon counts vs energy distribution curves at various tube voltages. The plot is for data collected using 100 micron collimator with the CdTe detector spectrometer over a 300 second time interval.

Figure 7 shows the ratio of alkali halide microstructured optical fiber to CdTe counts as a percent efficiency vs tube voltage. The $\mathrm{CsI}(\mathrm{Tl})$ fiber output exceeds the CdTe detector for all energies. This is because approximately 620 photons are emitted for each $10 \mathrm{keV}$ x-ray absorbed, as shown in Table 2. The uncoated NaI(Tl) and CsI(Na) fibers show a similar trend to the $\mathrm{CsI}(\mathrm{Tl})$ fiber. The $\mathrm{NaI}(\mathrm{Tl})$ fiber is over $170 \%$ efficiency at $10 \mathrm{kV}$ and over $70 \%$ at $40 \mathrm{kV}$ tube voltages. The CsI(Na) fiber is over $50 \%$ efficient at $10 \mathrm{kV}$ and over $30 \%$ at $40 \mathrm{kV}$ tube voltages. These efficiencies are dependent on the detector efficiency, shown in Table 3. With higher quantum efficiency detectors in the $420 \mathrm{~nm}$ wavelength range, the $\mathrm{CsI}(\mathrm{Na})$ and $\mathrm{NaI}(\mathrm{Tl})$ fibers output would exceed that of the CdTe detector.

Applying an external thin film coating of aluminum on these fibers resulted in a drastic reduction in detection efficiency. The aluminum coated fibers show significantly lower efficiencies with $\mathrm{CsI}(\mathrm{Tl})$ having the highest values over $20 \%$ at $10 \mathrm{kV}$ and over $5 \%$ at $40 \mathrm{kV}$ tube voltage. The $\mathrm{NaI}(\mathrm{Tl})$ and $\mathrm{CsI}(\mathrm{Na})$ efficiencies are a few percent as shown in Fig. 7.

This reduced efficiency is due to a change in the fiber optical characteristics from the aluminum coating. The fiber transmits less scintillation light when coated with a thin aluminum film when compared to being uncoated. The decrease in efficiency from the aluminum coating also demonstrates the experimental technique is sensitive to optical variations in the alkali halide fibers. In summary, the aluminum coating was applied as a means of protecting the alkali halide fibers from moisture and adds strength, to observe the optical effects of a reflective thin film on the efficiency, and provided a way to observe the sensitivity of the experimental setup to optical variations.

Based on the orders of magnitude greater number of photons emitted by alkali halide scintillators when compared to the number of $\mathrm{x}$-rays, it is possible that even greater efficiencies can be attained with similar optical fibers. However, the fiber optics must be carefully considered at the alkali halide emission wavelengths. Additionally, the crystal growth process directly affects the fiber output. 
In this work, improvements could be made with a photon detector having a quantum efficiency of $50 \%$ in the $420 \mathrm{~nm}$ wavelength range so the $\mathrm{NaI}(\mathrm{Tl})$ measured efficiency would be doubled and exceed that of the $\mathrm{CsI}(\mathrm{Tl})$ fiber. The CsI $(\mathrm{Na})$ fiber efficiency would be improved with a similar higher quantum efficiency detector. Optimizing the crystal growth conditions can also improve the measured efficiency.

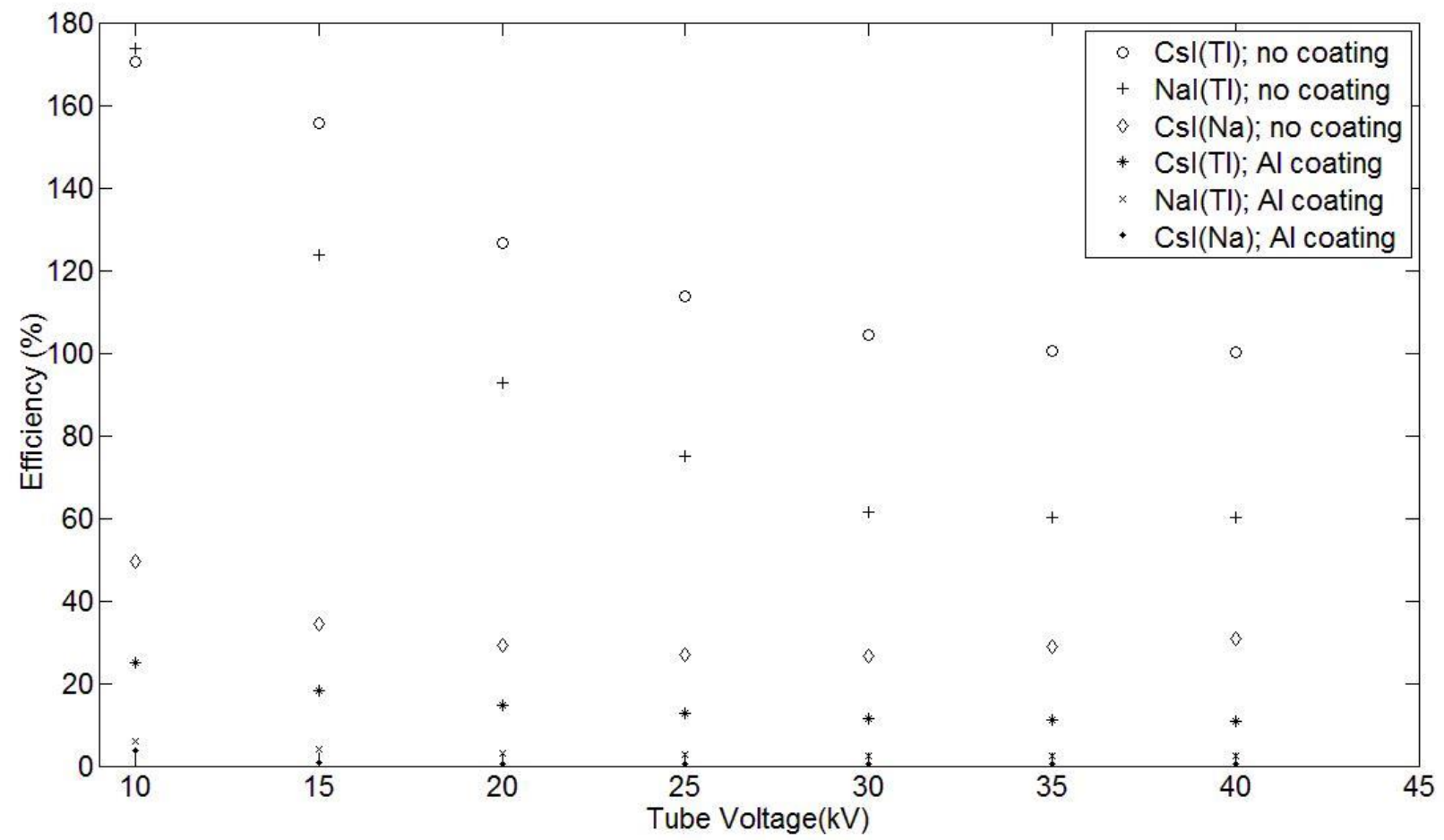

FIGURE 7. Shown is a plot of x-ray efficiency for fibers at tube voltages from 10 to $40 \mathrm{kV}$. The CsI(Tl) fiber efficiency is over $100 \%$ at all tube voltages. Addition of aluminum on the fiber exterior causes a change in the optical characteristics and a significant drop in efficiency.

\section{REFERENCES}

1. G. F. Knoll, Radiation Detection and Measurement (John Wiley \& Sons, NY, NY, 2000), pp. 219-262.

2. P.A. Rodnyi Physical Processes in Inorganic Scintillators (CRC Press, NY, NY, 1997), pp. 1-51.

3. H. Leutz, "Scintillating Fibers", Nucl. Instr. and Meth. 364, 442-448 (1995).

4. S.L. DeHaven, "Microstructured optical fiber for x-ray detection" Rev. Prog. QNDE 29 AIP Conf. Proc. 1121 541-548 (2009).

5. S.L. DeHaven, S. Albin, and W. Kelliher, "Liquid filled microstructured optical fiber for X-ray detection" Opt. Express 18, 13754-13760 (2010).

6. L. Hammar, "Novel design of high resolution imaging x-ray detectors" in $18^{\text {th }}$ World Conference on Nondestructive Testing, April 16-20, Druban, South Africa (2012).

7. NIST Website: Tables of X-Ray Mass Attenuation Coefficients and Mass-Energy Absorption Coefficients, http://physics.nist.gov/PhysRefData/XrayMassCoef/cover.html. 